\title{
La maternidad y el trabajo en Chile: Discursos actuales de actores sociales ${ }^{1}$
}

\author{
Elisa Ansoleaga
}

Universidad Diego Portales, Santiago, Chile. Email: maria.ansoleaga@udp.cl

\section{Lorena Godoy}

Universidad Diego Portales, Santiago, Chile. Email: lorena.godoycatalan@gmail.com

Resumen: La relación entre maternidad y trabajo ha sido materia de políticas públicas desde comienzos del siglo XX, pero en las últimas décadas ha adquirido nuevas connotaciones por el aumento de la participación laboral femenina. Ello no ha conducido a una eliminación de las discriminaciones que enfrentan las trabajadoras por su condición (real o potencial) de madres, ni a una organización del mercado de trabajo que permita articular demandas laborales y familiares, ni una redistribución del trabajo reproductivo. Para analizar cómo actores involucrados en la formulación de políticas relacionadas con esta temática concebían la relación entre trabajo y maternidad y las tensiones que derivaban de ella, se entrevistó a un grupo de actores sociales provenientes de tres ámbitos. Dos ideas caracterizan sus concepciones: las normas de protección a la maternidad en el trabajo afectan negativamente la empleabilidad femenina y la relación maternidad trabajo es difícil porque la organización del mercado de trabajo dificulta la articulación de responsabilidades laborales y familiares. Respecto de las tensiones, se constataron en diferentes dimensiones: económica, demográfica, ejercicio de derechos y sociocultural.

Palabras clave: maternidad, trabajo, salud, actores sociales.

\section{Motherhood and work in Chile: Current discourses of social actors}

\begin{abstract}
The relationship between motherhood and work has been a public policy topic since the early twentieth century, but in recent decades this relation has taken on new connotations due to the increase in female labor force participation. This has not led to an elimination of the discrimination of female workers, because of their status as real or potential mothers, nor to an organization of the labor market that allows harmonizing work and family demands, nor to a redistribution of reproductive work. To analyze how actors involved in policy formulation face this issue, how they understand the relationship between work and motherhood and the tensions that arise from it, a group of social actors from three areas were interviewed. Two ideas characterize their conceptions: the rules of maternity protection at work affect adversely the employability of women; and the relationship work-motherhood is hard because the organization of the labor market makes it difficult to articulate work and family responsibilities. Regarding tensions, they were identified in different areas: economic, demographic, socio-cultural and exercise of rights.
\end{abstract}

Key words: motherhood, work, health, social actors. 


\section{Maternidade e trabalho no Chile: Discursos atuais dos atores sociais}

Resumo: A relação entre maternidade e trabalho tem sido a política pública desde o início do século XX, mas, nas últimas décadas adquiriu novas conotações para o aumento da participação do trabalho feminino. Isso não levou a uma eliminação da discriminação enfrentada pelas trabalhadoras, devido à sua condição de mães (real ou potencial), ou a uma organização do mercado de trabalho para articular as demandas do trabalho e da família, ou uma redistribuição do trabalho reprodutivo. Para analisar como os atores envolvidos na formulação de políticas relativas a este assunto compreendem a relação entre trabalho e maternidade e linhagens derivadas dela, reuniu-se um grupo de atores sociais a partir de três áreas. Dois pensamentos caracterizam suas concepções: a legislação de protecção da maternidade no trabalho afeta negativamente a empregabilidade feminina ea relação trabalhos e maternidade é difícil, porque a organização do mercado de trabalho dificulta a articulação do trabalho e responsabilidades familiares. Quanto as tensões, foram encontrados em diferentes dimensões: econômica, demográfica, exercício dos direitos e sociocultural.

Palavras-chave: maternidade, trabalho, saúde, atores sociais.

$* * *$

\section{Antecedentes}

La relación entre maternidad y trabajo ha sido materia de debate y objeto de políticas públicas desde comienzos del siglo XX en Chile.

Desde el ámbito del trabajo, medidas de protección a la maternidad tienen una larga data (1917). Aunque inicialmente estas medidas fueron resistidas -invocándose los costos que implicaban para el empleador-, lograron generar un importante consenso entre diversos actores sociales respecto de la necesidad de proteger a las madres obreras -consideradas símbolo de los peores excesos del capitalismo industrial- para reducir los perniciosos efectos que el desempeño de ciertas actividades remuneradas podían tener para sus capacidades reproductivas (Hutchison, 2006). A juicio de Casas y Valenzuela (2011), parte importante de esta legislación de protección a la maternidad en el trabajo se ha dirigido predominantemente a resguardar la infancia y la familia y no necesariamente ha conducido a un mejoramiento de las condiciones de trabajo de las madres.

Desde el ámbito de la salud, la atención a la madre y al niño constituyó durante gran parte del siglo XX uno de los problemas de salud pública prioritarios concentrando parte importante de las atenciones y del presupuesto del sistema de salud. Como lo señala Zárate (2008), a comienzos de siglo la asistencia al parto fue una materia pública que excedió la frontera del ámbito clínico para ser también uno de los ejes de la cuestión social. A mediados de siglo, en el marco de la creación del Servicio Nacional de Salud, la atención materna infantil pasó a ser uno de los programas más importantes (Zárate \& Godoy, 2011). Alvarado y Herrera (2011) señalan 
que gran parte de las políticas de salud dirigidas a madres y niños, se centraron fundamentalmente en la protección a la primera infancia. Solo recientemente, desde los años noventa en adelante, se incorpora una perspectiva de género en el concepto de la salud de la mujer y de las políticas públicas dirigidas a ellas, lo cual ha permitido ampliar el alcance de la protección en salud más allá de la maternidad e incorporar aspectos ligados a la salud de las mujeres en distintas etapas de su ciclo de vida (Alvarado \& Herrera, 2011).

En las últimas décadas del siglo XX, la relación entre maternidad y trabajo adquirió nuevas connotaciones a la luz de una serie de importantes transformaciones. El aumento del número de mujeres que se integraron al mercado de trabajo, el crecimiento más rápido de sus tasas de ocupación en relación a las de los hombres, el aumento de sus años activos así como su presencia en empleos técnicos y profesionales. En este sentido, la inserción laboral femenina se volvió más numerosa, más permanente, más diversificada y con mayores niveles de educación (Godoy, Díaz \& Mauro, 2009).

Este aumento del empleo femenino ha ido acompañado de una valoración social del trabajo remunerado de las mujeres muy positiva por las oportunidades que brinda a las mujeres para incrementar los niveles de autonomía económica y libertad personal, y por su importante aporte a la reducción de la pobreza. En este contexto, contrario a lo sucedido hacia principios y mediados de siglo pasado, las políticas públicas han buscado incentivar el ingreso de mujeres al mercado de trabajo (Godoy, Díaz \& Mauro, 2009).

Diversos factores permiten entender estos cambios en el comportamiento laboral de las mujeres. Las transformaciones del modelo capitalista taylorista-fordista a un modelo de capitalismo flexible, la mayor necesidad de los hogares de contar con más ingresos, las crisis económicas, la disminución de las tasas de fecundidad, el mayor acceso a la educación y cambios culturales que favorecen el ejercicio por parte de las mujeres de roles tradicionalmente asociados a los hombres (Abramo \& Valenzuela, 2006). Todo ello ha puesto en tensión ciertas concepciones de género, especialmente aquella que asume el trabajo productivo como una actividad asignada a los hombres.

Otro factor determinante alude a los profundos cambios en el ámbito de la familia y al crecimiento de hogares monoparentales. En Chile, las jefaturas de hogar urbanas femeninas pasaron de un $24 \%$ en 1990 a un 32\% en el 2007, lo que equivale a un tercio de los hogares en los cuales las mujeres tienen a su cargo la provisión del sustento económico. Esto conlleva un sinnúmero de consecuencias en lo que refiere a atender las tareas de cuidado y el sustento económico familiar simultáneamente, entre ellas, que las jefas de hogar dejan a niños pequeños, ancianos o discapacitados sin atención para salir a trabajar; que las hijas mayores son retiradas del sistema escolaridad para cubrir estas tareas; y que una proporción importante 
de mujeres jefas de hogar entran en una inactividad optativa, o bien acceden a trabajos informales o de tiempo parcial que en la mayoría de los casos son mal remunerados y en condiciones precarias (OIT-PNUD. 2009).

Los significativos cambios en la distribución del trabajo productivo que los fenómenos señalados han provocado -y sus implicancias en las relaciones e identidades de género-, no han sido acompañados de cambios en la distribución del trabajo reproductivo (labores domésticas y trabajo de cuidado). Como lo indican diversos estudios, independientemente de su condición laboral, las mujeres destinan más tiempo que los hombres a las tareas de cuidado y a las labores domésticas; y los hombres, con independencia de su condición laboral, destinan menos tiempo que las mujeres a dichas labores (Godoy, Díaz \& Mauro, 2009). Esto se observa en diversos países de la Latinoamérica. En Chile, para el año 2007 los hombres destinaban 18 horas semanales a los quehaceres del hogar mientras las mujeres 28 horas semanales. Similar brecha se observa en Uruguay para el mismo año (13 vs. 35) en tanto lo reportado por México en el 2002 era de 10 vs. 50 (OIT. 2011).

Se asiste entonces a una fuerte contradicción entre las políticas de incentivo al empleo femenino -y la mayor disposición de mujeres a ingresar al mercado laboral- y: una organización del mercado de trabajo que dificulta seriamente la articulación de las responsabilidades laborales con la maternidad y el cuidado infantil; la persistencia de una determinada distribución sexual del trabajo que mantiene principalmente en sus manos el trabajo reproductivo; y la permanencia de procesos de discriminación laboral por razones de género que afectan a las trabajadoras relacionados, en parte importante, con su condición real o potencial de madres.

En este sentido, la actual relación entre maternidad y trabajo afecta en forma negativa las posibilidades de inserción laboral de las mujeres y las condiciones en las que ocurre dicha inserción; y determina una importante sobrecarga para las trabajadoras que deben articular cotidianamente sus responsabilidades laborales con el trabajo doméstico y el cuidado infantil. Junto con ello, afecta sus posibilidades de desarrollar proyectos laborales, proyectos familiares y su salud física y mental.

Considerando estos antecedentes, un equipo interdisciplinario de investigadores de las Facultades de Psicología, Derecho y Medicina de la Universidad Diego Portales, llevaron a cabo un estudio que cuyo objetivo fue generar información sobre las tensiones que emergen en torno a la relación entre maternidad, trabajo y salud durante el siglo XX en Chile, y proponer acciones para una política pública que reconozca dichas tensiones a fin de mejorar la actuales condiciones de vida de las mujeres.

En el marco de este estudio, se realizó un análisis de los discursos actuales de actores sociales provenientes de distintos sectores de la sociedad, con participación en la discusión y formulación de políticas públicas relacionadas con la maternidad, el trabajo y la salud, y en el estudio de 
temas relacionados con dicha temática ${ }^{2}$. El objetivo de este análisis fue acceder a través de sus discursos al modo como concebían la actual relación entre trabajo y maternidad, sus creencias y opiniones al respecto; y a los problemas y tensiones que se derivaban de dicha relación de modo de identificar elementos que ayudaran a comprender la persistencia de dichas tensiones y las consecuencias de ellas en la vida de las mujeres ${ }^{3}$. De acuerdo con este objetivo, el artículo presenta los principales resultados y discusiones que surgen a partir del análisis de los discursos de actores sociales respecto de las tensiones en torno a la maternidad y el trabajo y las consecuencias en la vida de las mujeres ${ }^{4}$.

\section{Método}

El estudio realizado fue de naturaleza cualitativa, lo que permitió acceder con mayor profundidad, y desde las explicaciones de los propios participantes, a la forma como concebían la relación entre trabajo, maternidad y salud.

\section{Participantes}

La muestra fue no probabilística, intencionada y por conveniencia. Se trató además de una muestra diversa o de máxima variación, utilizada en la investigación cualitativa cuando se busca mostrar distintas perspectivas y representar la complejidad del fenómeno estudiado, o bien documentar la diversidad, localizar diferencias y coincidencias, patrones y particularidades (Hernández, Fernández \& Baptista, 2006).

Como criterios de inclusión de la muestra se definió la pertenecía de los entrevistados/as a tres grandes categorías:

- Especialistas o Expertos: Se incluyó a personas que debido a sus conocimientos y trayectorias han sido consultadas en diversos procesos de toma de decisiones de la política pública en torno a temas relativos a la maternidad, el trabajo y la salud. Varios de los/as entrevistadas pertenecientes a este grupo fueron parte de la Comisión de Protección de la Infancia y de la Comisión de Trabajo y Equidad, organizadas durante el Gobierno de Michelle Bachelet; y de la Comisión Presidencial Mujer, Trabajo y Maternidad, convocada durante el actual gobierno de Sebastián Piñera. Otros entrevistados/as fueron seleccionados/as por su producción académico/científica en torno al tema.

- Actores del Estado: Se incluyó a personas que se desempeñaban como ministros de gobierno (ex ministra del Trabajo) y a parlamentarios involucrados en el proceso de toma de decisiones en temáticas vinculadas a los objetivos del estudio (miembros de comisiones 
de trabajo y/o de salud de la Cámara de Diputados y del Senado).

- Actores de Organizaciones Sociales y de la Sociedad Civil: Se consideró a personas que pertenecen a una diversidad de organizaciones e instituciones (centros de estudio, personas influyentes en la opinión pública (líderes de opinión), asociaciones gremiales de empresarios, organizaciones de trabajadores,) cuyas opiniones son relevantes para ilustrar la heterogeneidad de posiciones que existen en el mundo social respecto de la relación entre maternidad, trabajo y salud.

La muestra definitiva quedó constituida por 17 personas distribuidas de la siguiente manera: 6 Especialistas, 5 Actores del Estado y 6 Actores pertenecientes a Organizaciones Sociales y de la Sociedad Civil.

\section{Instrumento}

La recolección de información se hizo a través de entrevistas individuales semi estructuradas, que permiten comprender la perspectiva de los propios participantes respecto del fenómeno estudiado, y tal como lo expresan con sus propias palabras (Taylor \& Bodgan, 1992).

El instrumento para la recolección de información consistió en una pauta de preguntas que contempló los siguientes temas:

- Relación entre maternidad, trabajo y salud. Características, principales actores involucrados (individuales, institucionales), bien superior que la legislación al respecto busca resguardar.

- Principales tensiones y problemas que surgen de la relación entre maternidad, trabajo y salud.

- Consecuencias de estas tensiones y problemas para la vida de los involucrados y para la sociedad.

\section{Procedimiento}

Las entrevistas se efectuaron entre octubre del 2010 y enero del 2011, se realizaron en Santiago y Valparaíso y tuvieron una duración aproximada de una hora. Fueron grabadas y transcritas en su totalidad. Todos los entrevistados contaron con la firma de una carta de consentimiento informado.

Es importante señalar que simultáneamente a la realización de las entrevistas, se llevaba a cabo una discusión pública acerca de un proyecto de ley de extensión del postnatal. Ello significó dificultades y oportunidades. En algunas entrevistas la gran visibilidad en los medios de comunica- 
ción de la discusión de este proyecto contribuyó a que lo declarado por algunos participantes se centrara fundamentalmente en este tema, en señalar su posición respecto de esta iniciativa gubernamental. En otros casos, la ventana política que abrió este proyecto de ley también permitió una mayor apertura e interés por el debate sobre estos asuntos y facilitó el acceso a importantes personalidades públicas relacionadas con la temática en estudio.

\section{Análisis de la información}

Las entrevistas fueron analizadas siguiendo los procedimientos metodológicos señalados por el análisis de contenido de tipo cualitativo, que permite efectuar inferencias válidas -sobre el autor, el mensaje, la audiencia- de un texto (Ruiz Olabuénaga, 2003, p. 199) para "descubrir la significación de un mensaje”. Se trata de un método que consiste en "clasificar y/o codificar los diversos elementos de un mensaje en categorías con el fin de hacer aparecer de la mejor manera el sentido" (Gómez, p. 2). En este caso se utilizó para identificar, la manera como los propios actores entrevistados describían la relación entre maternidad, trabajo y salud, las tensiones que emergían, las consecuencias y posibles alternativas de acción.

Para ello se realizó, en primer lugar una lectura de las entrevistas (texto de campo), distinguiendo unidades de registro, es decir, todo elemento formal de contenido presente en el texto que se relacionara con el fenómeno a estudiar.

En segundo lugar, se distinguieron categorías que aluden a aquellas temáticas importantes en torno a las cuales se puede reorganizar el material, por ejemplo, agrupando todos aquellos enunciados referidos a un mismo sentido en una categoría. Esta etapa de reorganización del material da paso a un texto de investigación. Las categorías fueron construidas considerando los objetivos del estudio así como lo que iba emergiendo del texto (Flick, 2002). Múltiples lecturas del texto permitieron la progresiva definición y precisión de las categorías, así como la identificación de las relaciones entre ellas que supusieron una mayor elaboración e integración de la información.

Las entrevistas transcritas en su totalidad fueron ingresadas al software ATLAS TI, donde se procedió a codificarlas de acuerdo a las categorías establecidas.

\section{Resultados}

A continuación se presentan los resultados del análisis realizado a las entrevistas referidos a al modo como los/as entrevistados/as concebían y describían la actual relación entre trabajo y maternidad, sus creencias y 
opiniones al respecto, y los problemas y tensiones que se derivaban de dicha relación.

De acuerdo con estos objetivos, y siguiendo los procedimientos señalados por el análisis de contenido de tipo cualitativo, se distinguieron diversas categorías que junto con considerar dichos objetivos tuvieron en cuenta nuevos contenidos que fueron surgiendo del relato de los entrevistados. Diversas lecturas permitieron precisar de mejor modo dichas categorías y establecer relaciones entre ellas. De acuerdo con este análisis se distinguieron dos grandes categorías en relación a la descripción de la relación entre maternidad y trabajo que se relacionan estrechamente entre sí, y en torno a las cuales fue posible agrupar una serie de otras categorías que emergieron del análisis (Tabla 1).

\section{Tabla 1. Categorías}

\begin{tabular}{|c|c|c|}
\hline \multirow{2}{*}{$\begin{array}{l}\text { Definición de la } \\
\text { relación maternidad, } \\
\text { trabajo y salud }\end{array}$} & \multicolumn{2}{|c|}{$\begin{array}{l}\text { Normas de protección a la maternidad en el trabajo afectan negativamente } \\
\text { la empleabilidad de las mujeres. }\end{array}$} \\
\hline & \multicolumn{2}{|c|}{$\begin{array}{l}\text { Relación entre matemidad y trabajo es una relación dificil y compleja pues } \\
\text { la organización del mercado laboral dificulta la articulación entre trabajo } \\
\text { productivo y responsabilidades familiares. }\end{array}$} \\
\hline \multirow[t]{10}{*}{$\begin{array}{l}\text { Tensiones y } \\
\text { problemas que } \\
\text { derivan de esta } \\
\text { relación maternidad, } \\
\text { trabajo y salud }\end{array}$} & Dimensión económica & $\begin{array}{l}\text { Desincentivo de la contratación femenina vs. } \\
\text { Discursos pro crecimiento económico que } \\
\text { incentivan ingreso de mujeres al mercado de } \\
\text { trabajo }\end{array}$ \\
\hline & \multirow[t]{3}{*}{$\begin{array}{l}\text { Dimensión de acceso } \\
\text { y ejercicio de } \\
\text { derechos }\end{array}$} & $\begin{array}{l}\text { Beneficios de contar con legislación como un } \\
\text { derecho conquistado vs. efectos discriminatorios } \\
\text { de esta legislación para trabajadoras }\end{array}$ \\
\hline & & $\begin{array}{l}\text { Beneficios de contar con legislación como un } \\
\text { derecho conquistado vs. factor de desigualdad } \\
\text { entre trabajadoras. }\end{array}$ \\
\hline & & $\begin{array}{l}\text { Empleo femenino como derecho y expresión de } \\
\text { equidad social y de género vs. Mercado laboral } \\
\text { que discrimina a las mujeres por razones de } \\
\text { género. }\end{array}$ \\
\hline & \multirow[t]{2}{*}{$\begin{array}{l}\text { Dimensión } \\
\text { demográfica }\end{array}$} & $\begin{array}{l}\text { Fomentar el empleo femenino vs. Aumentarlas } \\
\text { tasas de natalidad. }\end{array}$ \\
\hline & & $\begin{array}{l}\text { Fomentar el empleo femenino vs. desincentivo a } \\
\text { la maternidad. }\end{array}$ \\
\hline & \multirow[t]{2}{*}{ El papel del Estado } & $\begin{array}{l}\text { Beneficios de contar con legislación de protección } \\
\text { a la maternidad vs. debates en torno a sistemas de } \\
\text { financiamiento de dicha legislación }\end{array}$ \\
\hline & & $\begin{array}{l}\text { Bien a proteger: derechos de los niños vs. Bien a } \\
\text { proteger derechos de las mujeres en tanto } \\
\text { trabajadoras y ciudadanas. }\end{array}$ \\
\hline & \multirow[t]{2}{*}{$\begin{array}{l}\text { Dimensión } \\
\text { Sociocultural }\end{array}$} & $\begin{array}{l}\text { La maternidad como un referente identitario } \\
\text { femenino vs. Diversificación/flexibilización de } \\
\text { referentes identitarios de género (papel del trabajo } \\
\text { remunerado) }\end{array}$ \\
\hline & & $\begin{array}{l}\text { Valoración de la maternidad vs. Falta de } \\
\text { condiciones materiales, laborales, de acceso a la } \\
\text { salud }\end{array}$ \\
\hline
\end{tabular}




\section{Definición de la relación entre maternidad y trabajo}

Respecto de la concepción de los entrevistados de la relación entre maternidad y trabajo, hay dos categorías.

En primer lugar -y corroborando lo que arroja el análisis de la legislación laboral sobre el tema (Casas \& Valenzuela, 2011)- la idea de que las normas de protección a la maternidad en el trabajo afectan negativamente la empleabilidad de las mujeres. Con independencia del grado de acuerdo que los actores consultados tengan respecto de estas normas, ellos indican que esta normativa puede desincentivar o dificultar la contratación de mujeres, encarecerla, determinar malas condiciones de trabajo y ser motivo de discriminación en los lugares de trabajo.

Actores más cercanos a sectores empresariales y políticamente conservadores (algunos entrevistados de Organizaciones Sociales y de la Sociedad Civil), consideran que la legislación de resguardo a la maternidad en el trabajo es excesiva lo que encarece la contratación femenina. Respecto de los costos asociados a dicha contratación, se mencionan como costos directos los asociados a la mantención de salas cunas y eventuales reemplazos. En tanto entre los costos indirectos se indica el fuero maternal -porque restringe la libertad de los empleadores para administrar sus recursos humanos-, y las licencias por enfermedades de hijos menores de un año -pues introducen una fuerte incertidumbre en el manejo de los recursos humanos y pueden desorganizar los procesos de trabajo obligando a hacer ajustes y reorganización de funciones sin previsibilidad. En definitiva, como lo afirma un parlamentario entrevistado, se trata de situaciones que alteran "el flujo natural del capital” (Parlamentario socialista).

Pero además de “encarecer” la contratación de mujeres, una idea muy recurrente es que la condición real o potencial de madres de las trabajadoras constituye uno de los factores más importantes en la determinación de malas condiciones de trabajo para ellas: menores salarios, menores posibilidades de promoción, malos tratos en el trabajo y mayor informalidad, condición esta última que se asocia con una precaria situación en materia previsional para las trabajadoras, y en limitaciones para acceder a las políticas de resguardo a la maternidad en el trabajo.

La segunda categoría es que la relación entre maternidad y trabajo, en un contexto laboral como el actual, es una relación compleja. Ello porque, la organización de las jornadas de trabajo, la intensificación del trabajo, los niveles salariales, la disponibilidad que se demanda a los trabajadores, etc., dificulta seriamente las posibilidades de articular familia y trabajo, convirtiendo dicha tarea en una cuestión difícil y desgastante, especialmente para aquellas personas que junto con el trabajo productivo tienen en sus manos el trabajo doméstico y de cuidado; personas que como sabemos siguen siendo predominantemente mujeres.

Ello trae consigo una serie de consecuencias negativas para las tra- 
bajadoras pues el esfuerzo cotidiano por lograr articular sus responsabilidades laborales y familiares se traduce en desgaste y cansancio permanente, y en una vivencia muy tensa de sus roles de madres y trabajadoras. Como señalan varios especialistas, parlamentarios y personas de organizaciones de la sociedad civil, algo que caracteriza la experiencia de la maternidad de mujeres trabajadoras (además del cansancio y desgaste) es la culpa. Algunas especialistas afirman que "las mujeres se sienten trabajadoras culpables, como que ellas están poniendo un peso en el lugar de trabajo a través de la maternidad"; "se sienten culpables de hacer valer las licencias de maternidad"; y al mismo tiempo, se sienten "madres culpables" por dejar a sus hijos por el trabajo (Especialista).

Es interesante mencionar lo que afirma un parlamentario entrevistado respecto que los problemas que ponen de manifiesto las madres trabajadoras, las dificultades para articular trabajo y familia, así como las malas condiciones laborales que enfrentan, son vistas como una expresión de "condiciones objetivas vinculadas a las mujeres, y no como un mercado laboral que les es hostil” (Parlamentario Socialista). Es decir, no se trataría de una organización del mercado de trabajo problemática, sino de personas problemáticas, en este caso mujeres que son madres o que eventualmente pueden llegar a serlo.

Esta forma de concebir la relación entre maternidad y trabajo refleja una serie de concepciones de género respecto de los espacios sociales y roles propios de hombres y mujeres, que en gran medida siguen sustentadas y se expresan en una distribución sexual del trabajo reproductivo (femenino). Ello explica en medida importante las tensiones y problemas que genera la actual relación entre maternidad y trabajo, los que se discuten a continuación.

\section{Tensiones y problemas que derivan de la relación maternidad, trabajo y salud}

En una dimensión económica, la contratación de mujeres resulta más costosa y es desincentivada por la legislación de protección a la maternidad cuestión que entra en tensión con discursos pro crecimiento económico y aumento de la productividad, en el marco de los cuales el fomento al empleo femenino ocupa un lugar importante. ¿Cómo se incentiva el ingreso de las mujeres al mercado de trabajo -reconociendo que se trata de trabajadoras cada vez más calificadas, que presentan capacidades especialmente adecuadas para desempeñar ciertos trabajos-, con una legislación de protección a la maternidad que obliga a los empleadores a asumir el costo de las salas cunas o que limita la libertad para disponer de este recurso en determinados periodos de tiempo (fuero maternal)?

Las tensiones en el plano económico adquieren gran relevancia pues en el discurso de actores entrevistados se advierte un claro predominio de 
una racionalidad económica en todo el debate sobre la legislación de protección a la maternidad en el trabajo. Se puede advertir con claridad cómo el lenguaje económico permea el discurso de muchos actores provenientes de distintos sectores profesionales, empresariales, sociales o políticos. Así, para referirse a la relación tensionada entre maternidad y trabajo, para definir qué medidas deben ser implementadas o cuales modificadas, se alude frecuentemente a análisis del tipo costo-beneficio.

Esta notoria gravitación de la racionalidad económica al momento de debatir respecto de la protección de la maternidad es fuertemente criticada por un parlamentario entrevistado, médico de profesión. Él afirma que diversos criterios para discutir los derechos de los trabajadores, y más específicamente los criterios médicos en relación a la protección a la maternidad, terminan siendo subordinados a los intereses del mercado. Lo que predomina es un criterio empresarial que permea a distintos actores sociales, y al cual debe supeditarse incluso la mayor evidencia científica con la que se cuenta hoy en relación, por ejemplo, a los beneficios de lactancia materna o el apego en la salud futura de niños y niñas.

Es decir, así como a comienzos y mediados del siglo pasado en la instalación de los derechos de protección a la maternidad primó el criterio médico -siendo determinante la influencia que ejercieron las bancadas médicas en la discusión parlamentaria- hoy dicho criterio es desplazado por el predominio del criterio económico. Criterio que no es de uso exclusivo de los economistas o empresarios sino que se instala en el lenguaje cotidiano de diversos actores sociales relevantes. El predominio de este lenguaje parece ilustrar el poder de una racionalidad económica por sobre otras lógicas y criterios, en la descripción o concepción de los problemas públicos, y por lo tanto, en la definición de políticas para abordar dichos problemas.

En una dimensión de acceso y ejercicio de derechos, se advierten varias tensiones. Una tensión entre los beneficios de contar con leyes de protección a la maternidad en el trabajo como un derecho conquistado, y los efectos discriminatorios para las mujeres trabajadoras que se le atribuyen a dicha legislación (malas condiciones de trabajo, limitaciones para la incorporación de mujeres en ciertos sectores y ocupaciones, entre otras).

Otra tensión se produce entre contar con esta legislación y el hecho que ella sea un factor que genere desigualdades entre las mujeres trabajadoras en la medida que el acceso a la protección a la maternidad o a las licencias por hijos menores de un año, está condicionado al tipo de contrato laboral de las trabajadoras, a su condición de formalidad o informalidad.

Otra tensión se genera entre el incentivo al empleo femenino concebido como un derecho, como expresión de una mayor equidad social y de género (y no sólo como un factor de desarrollo económico), versus un mercado laboral que sigue discriminando a las mujeres por razones de género. Ello configura en las mujeres una experiencia del trabajo productivo muy ambigua, pues al mismo tiempo que dicho trabajo puede ofrecer importan- 
tes oportunidades de desarrollo personal y profesional, y de aumentar sus niveles de autonomía personal y económica, constituye un espacio que las discrimina por el hecho de ser mujeres, y muy especialmente, por su condición real o potencial de madres.

Desde una dimensión demográfica, se produce una tensión entre fomentar el ingreso de las mujeres al mercado de trabajo y aumentar las tasas de natalidad. Es como si la inserción laboral de las mujeres, el éxito profesional y laboral, se viviera en la práctica como un desincentivo a la maternidad. Un entrevistado perteneciente a la sociedad civil, explica que la relación entre maternidad y trabajo se asemeja a "un juego, digámoslo así, de suma cero entre ambos fenómenos, o sea, conforme se estimula más el ingreso de las mujeres al mundo del trabajo, disminuye la propensión de las mujeres a la maternidad, o por lo menos aumenta la decisión de postergar la maternidad” (Líder de opinión y académico). Ello explicaría -además de la reducción del número de hijos- fenómenos como el aplazamiento de la maternidad a edades en las cuales las mujeres tienen carreras laborales más consolidadas.

En relación al papel del Estado y de las políticas públicas en estas materias, se plantean varias tensiones y problemas. Los beneficios de contar con una legislación que protege la maternidad en el trabajo suscitan tensiones en torno a los sistemas de financiamiento de esta normativa: debe ser asumido sólo por el Estado o en él debe concurrir también el sector privado.

Por otra parte, dado el carácter presidencialista de nuestra institucionalidad legislativa (el Ejecutivo propone proyectos de ley al Legislativo), los gobiernos se ven enfrentados a una tensión importante entre proponer políticas del corto plazo que sean capaces de realizarse en consideración de los ciclos políticos electorales (lógica de resultados en el corto plazo) o bien diseñar y proponer una política pública de más largo aliento bajo el concepto de una política de Estado que excede su permanencia en el poder (lógica de resultados en el largo plazo). Así, lo que determina el establecimiento de la agenda político-legislativa de un gobierno, más que un proyecto de sociedad o idea de país, son los ciclos políticoelectorales y las posibles ganancias asociadas a la determinación de una política pública.

El Estado tiene la cautela de ciertos bienes públicos y esta es otra cuestión problemática al momento de pensar en las políticas públicas de protección a la maternidad. ¿Cuál es el bien que se busca proteger a través de la normativa: acaso son los derechos de la infancia, de los niños/as, o son los derechos de las mujeres en tanto ciudadanas, en tanto trabajadoras? Algunos especialistas y actores pertenecientes a organizaciones de la sociedad civil, critican el hecho que gran parte de la legislación de protección a la maternidad en el trabajo tenga por propósito, como ha sucedido en décadas pasadas, la protección de los derechos de los niños/as y no los de las mujeres en tanto trabajadoras y ciudadanas ${ }^{5}$. 
Atendiendo a ello, se plantea la necesidad de balancear los derechos e intereses de todas las partes involucradas, mujeres y niños, y no tratarlos como cuestiones antagónicas: o se fomenta la contratación de mujeres o se fomenta la lactancia materna exclusiva como condición para el desarrollo adecuado de los niños/as. Ello porque se reconocen las estrechas vinculaciones que existen entre el bienestar de ambos, lo que hace necesario conjugar los intereses de niños/as y madres. Al respecto una especialista entrevistada afirma que el desarrollo de los niños debe armonizarse con los intereses de las madres: "si tenemos a una madre que no tiene sus derechos como trabajadora bien resueltos, vamos a tener un niño mal cuidado, maltratado" (Entrevista a Especialista, médico).

En una dimensión sociocultural, en el plano de las creencias y mandatos respecto de los campos sociales que constituyen las esferas principales de integración social de los hombres y las mujeres, y de los roles asignados a cada uno de ellos, se advierte que una serie de cambios ocurridos a nivel laboral, económico, educacional, cultural y familiar, ponen en tensión dichas creencias y mandatos de género. Es decir, se trata de un determinado orden de género que se ha visto fuertemente cuestionado por esta serie de cambios, entre los cuales uno de los más relevantes ha sido el aumento de la participación laboral femenina y la positiva valoración social que existe en la actualidad de dicha participación. Algunas expresiones de este orden tensionado serían las siguientes.

La mantención de la maternidad como un referente identitario femenino central, lo que contrasta con la diversificación de referentes de género, especialmente en el caso de las mujeres, entre los cuales el trabajo remunerado adquiere una notoria importancia. En efecto, y como se ha señalado, existe una positiva valoración del trabajo remunerado de las mujeres en tanto medio que permite una mayor autonomía económica y personal de las mujeres, y les ofrece posibilidades de realización personal y desarrollo laboral y profesional.

En las opiniones expresadas por los entrevistados es posible advertir estos contrastes. Así, hay entrevistados que valoran muy positivamente la inserción laboral de las mujeres como factor de autonomía y desarrollo, y que critican el predominio del resguardo de los derechos de los niños/as por sobre los de las mujeres en la legislación de protección a la maternidad en el trabajo. Otros actores entrevistados, junto con tener una positiva opinión de la inserción laboral de las mujeres por su contribución al desarrollo económico, conciben su trabajo remunerado como secundario, complemento o apoyo al ingreso principal del hombre, y mantienen una idea de que el trabajo doméstico y de cuidado es una responsabilidad de las mujeres.

Otra expresión de este orden de género cuestionado es el "doble discurso" que se produciría entre una valoración social y cultural de la maternidad, y al mismo tiempo la falta de condiciones materiales, laborales, dificultades para acceder a servicios de salud, para que madres trabajadoras puedan vivir adecuadamente su maternidad. 
Se trataría de un reconocimiento de la importancia social de la maternidad, especialmente en un contexto de reducción de las tasas de natalidad, pero en la práctica no asumir socialmente todo lo que ello implica, dejando en manos de las mujeres las responsabilidades y los importantes costos asociados. Por ejemplo, en el ámbito del trabajo los costos del cuidado infantil (salas cunas) se cargan a ellas, lo que como afirman algunos entrevistados genera desincentivos o trabas a la contratación de mujeres y determina condiciones de trabajo menos favorables para ellas.

\begin{abstract}
“(...) son los mismos sectores [de empresarios que ven la contratación de mujeres como un problema] los que, a su vez, por otro lado, podrían hacer hermosas declaraciones sobre la importancia de la familia, la maternidad, entre otras cosas. En resumen, yo creo que este doble discurso social sobre la maternidad y muy manejado desde la emoción, se estrella con la realidad que uno vive, que uno ve, que uno escucha de las mujeres que tienen que buscar esas atenciones” (Especialista entrevistado, médico).
\end{abstract}

En el ámbito del acceso a la salud, este discurso que ensalza la maternidad se 'estrella' con el encarecimiento de los planes de salud para mujeres en edad fértil porque como lo afirma el mismo médico entrevistado, se concibe el embarazo como un siniestro: “en la lógica de los seguros, es decir, el embarazo es un siniestro, y si usted quiere tener hijos, usted los paga, sola” (Especialista entrevistado, médico).

En definitiva lo que este doble discurso expresa es una valoración de la maternidad pero la ausencia de condiciones materiales concretas -en términos de organización del mercado de trabajo, de redistribución de tareas reproductivas (cuidado y domésticas) entre hombres y mujeres al interior de las familias, de organización de sistemas de cuidado, de acceso a sistemas de salud-, que asuman en los hechos la reproducción como un asunto que atañe a toda la sociedad, y no como un asunto de las mujeres. En la práctica se sigue actuando, más allá de ciertos discursos que plantean que la maternidad y el cuidado de los hijos debe ser asumido como un asunto de la sociedad que involucra a diversos actores, como si se tratara de una cuestión de las mujeres que enfrentan fundamentalmente con estrategias individuales o familiares.

\title{
Reflexiones Finales
}

A partir del análisis de los discursos de los actores sociales entrevistados, presentamos algunas reflexiones finales.

Respecto de la importancia de la idea de los efectos negativos de la legislación de protección a la maternidad en el trabajo, en la empleabilidad de las mujeres, nos parece importante puntualizar que la maternidad real o potencial de las trabajadoras mujeres, junto con constituirse en una barrera 
de entrada al mercado de trabajo -como se desprende de lo señalado por algunos actores consultados- es un factor importante en la determinación de malas condiciones de trabajo para las mujeres. Es decir, más que impedir el ingreso de mujeres, incide en que este ingreso se haga en malas condiciones: menores salarios, menores posibilidades de promoción, malos tratos y mayor informalidad.

Ello no significa negar que la maternidad en determinadas ocupaciones y sectores productivos, sí pueda tener el efecto de barrera de entrada para las mujeres. Lo que hace que en ciertas circunstancias la maternidad real o potencial de las mujeres sea utilizada o no como un argumento para decidir la contratación de mujeres, es el carácter de género -masculino o femenino- atribuido a determinadas ocupaciones. Como se puede apreciar en el mercado laboral actual (ocupaciones feminizadas; ocupaciones masculinizadas), como lo han indicado anteriores estudios y como lo ratifican algunas de las trabajadoras madres que fueron consultadas en el marco de este mismo estudio, el carácter femenino atribuido a una ocupación puede pesar mucho más que la condición real o potencial de madres de las trabajadoras, a la hora de decidir a quién contratar. Por lo tanto, los efectos apocalípticos que se le atribuyen a la legislación de protección a la maternidad sobre el ingreso de mujeres al mercado de trabajo, no necesariamente se concretan en importantes sectores económicos en que se ocupan mayoritariamente mujeres (servicios sociales y comunales; servicios educacionales y de salud, retail, entre otros). Se trata de un argumento -uno de los principales en contra de la legislación de protección a la maternidad en el trabajo y de las modificaciones actuales introducidas a la duración del postnatal-, que adolece de evidencia empírica que lo sustente y pareciera ser un argumento de índole más bien ideológica a favor del mercado y de los intereses empresariales.

Otra cuestión que interesa resaltar es la gravitación que sigue teniendo el argumento de que la contratación de mujeres es más cara, que sus costos laborales serían más altos, producto de la legislación de protección a la maternidad. Argumento que llama la atención pues existe escasa evidencia que avale esta afirmación. Como lo señalan Abramo y Todaro (2006), en América Latina existe escasa información sobre el tema desagregado por sexo en relación a los diversos factores que podrían incidir en los costos laborales, o no se encuentra disponible de manera regular. Y junto con ello, los estudios que se han realizado sobre el tema concluyen que los costos laborales de las mujeres o son inferiores a los de los hombres o las diferencias son mínimas ${ }^{6}$.

Aunque la relevancia de este tipo de argumentos se puede entender en un contexto como el actual en el que, como se señaló, la racionalidad económica es predominante al momento de argumentar y discutir las normas de protección a la maternidad en el trabajo (y se podría señalar, de normas de protección de los derechos de los trabajadores en un sentido más general); por lo mismo sorprende la escasez de estudios que avalen más empíricamente este tipo de afirmaciones. 
Por último, quisiéramos señalar que la falta de condiciones materiales mínimas que enfrentan muchas trabajadoras para asumir su maternidad, el doble discurso del que hablábamos, debe ser considerado para entender ciertos planteamientos que afirman que hoy las mujeres tendrían mayores opciones para desarrollar una carrera laboral y ser madres; planteamientos desde los cuales se asume que la menor cantidad de hijos o el aplazamiento de los embarazos obedecerían sólo a una “opción” de las mujeres.

Sin dejar de reconocer y valorar (por cierto) que efectivamente hoy una serie de cambios han contribuido a ampliar las posibilidades de inserción laboral de las mujeres, y a cuestionar que la maternidad sea el destino irrenunciable de ellas, las discriminaciones que siguen afectando a las mujeres en el mercado de trabajo relacionadas con su condición de madres, las dificultades que la organización del mercado de trabajo ofrece para articular las responsabilidades laborales con la vida familiar, los mayores costos de los planes de salud de las mujeres, son condiciones materiales que restringen seriamente la capacidad de opción de las mujeres.

En este sentido, deberíamos pensar en qué medida el aplazamiento de la maternidad o la reducción del número de hijos expresa no solo cambios culturales importantes y valorados, sino también esta falta de condiciones materiales en términos de organización del mercado de trabajo, de redistribución de tareas de cuidado y trabajo doméstico entre hombres y mujeres al interior de las familias, de organización de sistemas de cuidado, de acceso a sistemas de salud-, para que las mujeres trabajadoras puedan vivir satisfactoriamente su maternidad. 


\section{Notas}

${ }^{1}$ Este artículo presenta los resultados de un estudio desarrollado en el marco del proyecto de investigación "Mujer, Trabajo, Maternidad, Salud: Tensiones no resultas del Siglo XX y Propuestas para el Bicentenario”. Proyecto de Investigación, Fondos Bicentenario, Universidad Diego Portales. 2010-2011. El estudio reunió a académicos de las Facultades de Psicología, Medicina y Derecho. El informe final está disponible en http://www.udp.cl/ investigacion/repo_detalle.asp?id=108.

${ }^{2}$ El estudio contempló además un análisis de prensa sobre discursos en torno a la relación a la maternidad y trabajo, entre 1999 y 2011; análisis de los discursos de madres trabajadoras de distintos sectores ocupacionales; descripción y análisis de las políticas de protección a la maternidad en el ámbito laboral desde comienzos del siglo XX hasta la actualidad; análisis de las políticas de salud dirigidas a madres y niños, desde comienzos del siglo XX hasta la actualidad; análisis sobre la evidencia respecto de la lactancia materna y sus efectos en la salud de la madre y el niño; análisis sobre la salud mental en la relación maternidad y trabajo.

${ }^{3}$ Las entrevistas también abordaron las propuestas que planteaban algunos entrevistados para abordar la relación entre maternidad y trabajo, aspecto que no será abordado en este artículo.

${ }^{4}$ Agradecemos la participación en la realización y análisis de las entrevistas de Mariana Fagalde en una primera etapa del estudio, y de Fernando Silva.

${ }^{5}$ Ello confirmaría lo señalado por Casas y Valenzuela (2011) en relación a la legislación de protección a la maternidad en el trabajo, y Alvarado y Herrera (2011) en relación a las políticas de salud dirigidas a la maternidad, durante el siglo XX.

${ }^{6}$ Rosalba Todaro (2002) realizó un estudio de costos laborales utilizando información sobre remuneraciones y costos de mano de obra desagregada por sexo, proveniente de $1050 \mathrm{em}$ presas del país. La información fue solicitada por el Servicio Nacional de la Mujer (SERNAM) al Instituto Nacional de Estadísticas (INE) pues las Encuestas de Remuneraciones y Costos de Mano de Obra que realiza el organismo desde 1993 no estaba desagregada por sexo. La autora concluyó que el costo laboral total de las mujeres, que incluye los costos salariales y los costos no salariales, alcanza el 68,4\% del costo laboral total de los hombres. Esta diferencia a favor de los hombres no solo se debe a que las remuneraciones de las mujeres eran inferiores a las de los hombres sino también a que eran menores los beneficios no salariales de las mujeres. Ello demostraba la poca incidencia que tenían los costos de salsa cunas o de los permisos de alimentación de los hijos, lo cual podía deberse a la baja tasa de fecundidad de las mujeres trabajadoras, el incumplimiento o no uso de este derecho por parte de las trabajadoras. Otro estudio realizado por OIT con datos de asalariados de Argentina, Brasil, Chile, México, Uruguay (1999-2000) (Abramo \& Todaro, 2006) indica que los costos monetarios para el empleador relacionados a la contratación de las mujeres son muy reducidos, representaban el $2 \%$ de la remuneración bruta mensual de las mujeres. Ello se debía a la baja incidencia anual de embarazos entre trabajadoras asalariadas, pero especialmente al hecho que las prestaciones monetarias asociadas a la maternidad no eran financiadas por los empleadores. Además, las prestaciones monetarias que recibían las mujeres durante la licencia-maternidad representaba menos del $2 \%$ de la masa salarial femenina registrada. Otros costos relativos a la lactancia (1\% de la remuneración bruta mensual de las mujeres) 
Polis, Revista Latinoamericana, Volumen 12, $N^{\circ}$ 35, 2013

y costos de reemplazo de una mujer durante la licencia de maternidad $(0,1 \%$ de la remuneración bruta mensual de las mujeres) también eran muy bajos. 


\section{Bibliografía}

Abramo, L. \& Todaro, R. (2006), “Costos laborales y reproducción social en América Latina”. En, Abramo, L. (Ed.) Trabajo decente y equidad de género en América Latina, OIT, 316 pp, Santiago de Chile.

Alvarado, N. \& Herrera, T. (2011), "Políticas Públicas de salud desarrolladas en Chile durante el último siglo”. En Mujer, Trabajo, Maternidad, Salud. Tensiones no resueltas del siglo XX y propuestas para el Bicentenario. Informe Final. Universidad Diego Portales. Santiago. Disponible en http://www.udp.cl/investigacion/repo_detalle.asp?id=108.

Casas, L. \& Valenzuela, E. (2011), “De la protección de la primera infancia a la protección de la familia”. En Mujer, Trabajo, Maternidad, Salud. Tensiones no resueltas del siglo XX y propuestas para el Bicentenario. Informe Final. Universidad Diego Portales. Santiago. Disponible en http:/ /www.udp.cl/investigacion/repo_detalle.asp?id=108.

Comisión Económica para América Latina y el Caribe [CEPAL] (2008), Anuario Estadístico de América latina y el Caribe 2007, CEPAL, Santiago de Chile.

Gómez Mendoza, Miguel Ángel (2000), “Análisis de contenido cualitativo y cuantitativo: Definición, clasificación y metodología”. Revista de Ciencias Humanas-UTP, mayo. Extraído el 3 de octubre de 2011, de: http:// www.utp.edu.co/ chumanas/revistas/revistas/rev20/gomez.htm

Hernández, R. Fernández, C. \& Baptista, P. (2006), Metodología de la Investigación. McGraw-Hill, México.

Hutchison, E. (2006), Labores apropiadas a su sexo. Género, políticas y trabajo en Chile urbano, 1900-1930, Editorial LOM, Santiago de Chile. 321pp.

Organización Internacional del Trabajo [OIT] (2011), Década del Trabajo Decente en las Américas: 2006-2015. Primer Balance y Perspectivas de la Agenda Hemisférica, OIT, Santiago de Chile.

Organización Internacional del Trabajo [OIT] - Programa de las Naciones Unidas para el Desarrollo [PNUD] (2009), Trabajo y Familia: Hacia nuevas formas de conciliación con corresponsabilidad social, OIT, Santiago de Chile.

Ruiz Olabuénaga, J. I. (2003), Metodología de la investigación cualitativa, Universidad de Deusto, Bilbao.

Taylor, S. \& Bodgan, R. (1992), La entrevista en profundidad. En Introducción a los métodos cualitativos de investigación, Paidós, Barcelona. 
Todaro, R. (2002), "Encuesta de Remuneraciones y Costo de la mano de obra, Análisis por sexo”. SERNAM Documento de Trabajo, $n^{\circ} 75$. Santiago, SERNAM, 51 pp, Santiago de Chile.

Zárate, S. \& Godoy, L. (2011), “Madres y niños en las políticas del Servicio Nacional de Salud. Chile, 1952-1964”. Revista História, Ciências, Saúde - Manguinhos (en edición).

Zárate, S. (2008), “L’assistance clinique et sanitaire de l'accouchement: Trajectoire nationale et influences internationales, Santiago du Chili, 1900 1950”, Canadian Bulletin of Medical History, Vol. 25, N 1, pp. 193-224.

Recibido: 05.11.2011

Aceptado: 25.10.2012 